\title{
What Happens after the Puerperium? Analysis of "Late" Postpartum Readmissions in California
}

\author{
Brett C. Young1 ${ }^{*}$, Erin Madden², Allison S. Bryant ${ }^{3}$ \\ ${ }^{1}$ Beth Israel Deaconess Medical Center, Department of Obstetrics, Gynecology and Reproductive Biology, \\ Division of Maternal Fetal Medicine, Harvard Medical School, Boston, USA \\ ${ }^{2}$ Northern California Institute for Research and Education, San Francisco, USA \\ ${ }^{3}$ Massachusetts General Hospital, Vincent Department of Obstetrics and Gynecology, Division of Maternal \\ Fetal Medicine, Harvard Medical School, Boston, USA \\ Email: bcyoung@bidmc.harvard.edu, erinmaddenmph@gmail.com, abryant@partners.org
}

Received 29 December 2014; accepted 23 February 2015; published 27 February 2015

Academic Editor: Mairiga A. Garba, University of Maiduguri, Nigeria

Copyright (C) 2015 by authors and Scientific Research Publishing Inc.

This work is licensed under the Creative Commons Attribution International License (CC BY).

http://creativecommons.org/licenses/by/4.0/

(c) (i) Open Access

\begin{abstract}
Objective: Admissions to acute care hospitals represent a significant portion of healthcare utilization. Little is known regarding hospitalization in the first postpartum year beyond the traditional 6 weeks of the puerperium. We sought to investigate whether there are identifiable risk factors for hospital readmission during this time period. Study Design: We conducted a retrospective population-based study using all California birth records between 1999 and 2003. These records were linked with hospital discharge data for all admissions to California hospitals in the first 365 days after delivery. For women with a first birth during the study period, we assessed the likelihood of readmission to an acute care hospital between 42 and 365 days post-delivery. Univariate and multivariable logistic regression were used to determine risk factors for these "late postpartum" admissions. Results: Of 951,570 maternal birth admissions during the time period, 15,727 $(1.7 \%)$ women were admitted in the late postpartum period. Women with an early postpartum readmission, antepartum admission, extremes of maternal age, black race, diabetes, hypertension, early preterm delivery and cesarean delivery had higher rates of late postpartum readmission. Of women with an antepartum admission for gestational diabetes or pre-existing diabetes, $6.6 \%$ and $18.5 \%$ of these women experienced a late postpartum admission for a diabetes-related diagnosis. Conclusion: Hospital readmission rates in the first year postpartum, remote from delivery, are significant. Women are at a higher risk of requiring hospital admission in the first year postpartum with select demographics and pregnancy-related diagnoses.
\end{abstract}

\footnotetext{
${ }^{*}$ Corresponding author.
}

How to cite this paper: Young, B.C., Madden, E. and Bryant, A.S. (2015) What Happens after the Puerperium? Analysis of "Late" Postpartum Readmissions in California. Open Journal of Obstetrics and Gynecology, 5, 123-127. 


\section{Keywords}

\section{Diabetes in Pregnancy, Hypertension in Pregnancy, Hospital Readmission, Postpartum}

\section{Introduction}

Pregnancy and the postpartum period may be viewed as a "window of opportunity" for women's health and health care [1]. Pregnancy complications such as preterm birth, gestational diabetes and preeclampsia have been associated with chronic diseases later in life requiring hospitalization and higher utilization of health care resources [2]-[4]. At the same time, for many women, pregnancies represent times of increased access to and interaction with the health care system. Having the ability to assess women's health status during pregnancy and the puerperium may contribute to improved care and outcomes beyond the immediate reproductive period. While other studies have found the likelihood of immediate postpartum readmissions to be $1 \%-2 \%$, [5]-[7] there are little data on hospital readmissions after the traditional postpartum period from 42 - 365 days postpartum. It is unknown whether there are any identifiable risk factors during pregnancy and the immediate puerperium that are associated with hospital admission in the first postpartum year.

Prior studies on immediate postpartum readmissions and readmissions up to 6 months following delivery have focused primarily on the route of delivery as well as the diagnosis on admission requiring hospitalization [7] [8]. These studies have been limited in their assessment of obstetrical or demographic risk factors or whether antepartum, early postpartum readmission or the duration of any of these admissions correlates with hospitalization up to 1 year postpartum.

Hospital readmissions have significant economic implications and may account for up to $20 \%$ of all health care costs [9]. As pregnancy and the puerperium have previously been associated with a woman's future health [2]-[4], we hypothesized that an antepartum admission and any readmission during the puerperium would increase the risk for hospital readmission up to one year postpartum. We sought to determine the proportion and characteristics of women requiring hospital admission after the traditional postpartum period and up to one year after delivery, with particular interest in obstetrical risk factors.

\section{Materials and Methods}

We conducted a retrospective population-based study. Data from vital statistics records for all births in California between 1999 and 2005 are linked with hospital discharge data for all California hospital admissions from conception to 365 days after delivery by the California Office of Statewide Health Planning and Development. Hospital discharge data include summaries of abstracted discharge diagnoses and procedures. Women are assigned a unique identifier that allows for linkages across all births to the same woman within the state of California. The linkage process used to create the merged file is described elsewhere, with 99\% accuracy of data linkage [10]. Our analyses are restricted to women with a first birth between 1999 and 2003. We defined the "late postpartum period" as the time between 42 and 365 days post-delivery and the "early postpartum period" as the time between delivery and 41 days after delivery.

We calculated the proportion of women readmitted to an acute care hospital in California during this late postpartum period and used univariate and multivariable logistic regression to determine risk factors for these admissions. We also determined the primary indications for late postpartum readmissions, as coded at the time of admission. Our primary outcome was the incidence of late postpartum readmission. We assessed the association of the following maternal factors with the primary outcome: length of stay during the birth admission and a late postpartum admission, maternal age, the month prenatal care began, gestational age at delivery, antepartum admission, an immediate postpartum readmission, maternal education, private insurance, mode of delivery, twin gestation, year of delivery, type of hospital, and the presence of: placenta previa, preterm labor, pregnancy-related hypertension, diabetes mellitus, gestational diabetes, other pregnancy complications, postpartum hemorrhage, anesthesia complications, major puerperal sepsis, hysterectomy, ethnicity, rural county, geographic location within California (northern vs.southern county).

As a secondary analysis, we used univariate and multivariate logistic regression to establish whether an antepartum diagnosis of gestational diabetes, pre-existing diabetes, gestational hypertension or pre-existing hyper- 
tension was associated with a late postpartum readmission for either a related diagnosis or was associated with any indication for a late postpartum readmission. Analyses were performed using the SAS v9.2 statistical package (Cary, NC). Institutional approval was granted by the Massachusetts General Hospital Institutional Review Board and the California Health and Human Services Agency's Committee for the Protection of Human Subjects.

\section{Results}

There were 2,603,948 birth admissions during the study period; of these, 951,570 were first births and were included in analyses for this study. Of these primiparous women, 15,727 (1.7\%) were admitted 42 - 365 days postpartum. There were 69,744 (7.3\%) women admitted during the antepartum period and 10,088 (1.1\%) women admitted during the first 41 days postpartum. Further descriptive statistics are displayed in Table 1 . The most common primary indications for late postpartum readmissions were biliary disease (23.7\%), appendicitis (5.7\%), other pregnancy complications (5.5\%), pancreatitis (4.7\%) and mood disorders (4.6\%). The mean length of stay for any late postpartum admission was $4.0+8.1$ days.

The following risk factors were associated with an increased risk for late postpartum readmission: maternal age $>40$ years old and $<20$ years old, black race, diagnosis of diabetes or gestational diabetes, preterm delivery at $<28$ weeks gestation, cesarean delivery, antepartum admission and readmission during the first 41 days postpartum. Univariate and multivariate regression results are displayed in Table 2.

Given that antepartum admission was positively associated with late postpartum admission, we further evaluated the specific indication for antepartum hospitalization and subsequent late postpartum hospitalization. Antepartum admission for gestational diabetes increased the risk for subsequent late postpartum diabetes-related admission; $6.6 \%$ of these women were admitted during the late postpartum period for a diabetes related admission. Similarly, antepartum admission for pre-existing diabetes increased the likelihood of late postpartum admission for diabetes complications with $18.5 \%$ of these women admitted during the late postpartum period. Women admitted antepartum for pre-existing chronic hypertension had a statistically significant increased risk for late postpartum admission for hypertension-related complications although the incidence remained low at $0.3 \%$.

\section{Comment}

The first postpartum year is an important time period as health concerns uncovered during pregnancy may further manifest in the late postpartum period. Much work in the obstetrical literature [5]-[8] has focused on events immediately following delivery, whether during the initial postpartum hospitalization or rehospitalization during the early postpartum period. There are few known predictors of admission during the late postpartum period and whether there are any associations between such admissions and pregnancy-related health.

Table 1. Demographics study cohort $(\mathrm{N}=951,570)$.

\begin{tabular}{cc}
\hline Maternal age (years) & $25.5 \pm 6.4$ \\
Gestational age at delivery (weeks) & $39.3 \pm 2.5$ \\
Registration of prenatal care (months) & $2.3 \pm 1.5$ \\
White & $34.9 \%$ \\
Black & $5.6 \%$ \\
Mexican/Hispanic & $44.2 \%$ \\
Length of stay at birth admission (days) & $2.7 \pm 2.3$ \\
Pre-existing diabetes mellitus & $3947(0.4 \%)$ \\
Gestational diabetes mellitus & $35,329(3.7 \%)$ \\
Hypertensive disorders of pregnancy & $44,342(4.7 \%)$ \\
Preterm delivery (<37.0 weeks) & $94,822(10.0 \%)$ \\
\hline
\end{tabular}

Data displayed as mean \pm SD or as $\mathrm{N}(\%)$ as appropriate. 
Table 2. Risk factors for late (42 - 365 days) postpartum admissions.

\begin{tabular}{ccccc}
\hline \multirow{2}{*}{ Variable } & \multicolumn{2}{c}{ Univariate Analysis } & \multicolumn{2}{c}{ Multivariate Analysis } \\
\cline { 2 - 5 } & OR [95\% CI] & p-value & aOR [95\% CI] & p-value \\
\hline Antepartum admission & $2.60[2.5-2.7]$ & $<0.0001$ & $2.13[2.0-2.2]$ & $<0.0001$ \\
Early postpartum admission (1 - 41 days) & $4.74[4.4-5.1]$ & $<0.0001$ & $3.86[3.6-4.2]$ & $<0.0001$ \\
Delivery $<$ 28 weeks gestation & $2.42[2.1-2.8]$ & $<0.0001$ & $1.42[1.23-1.65]$ & $<0.0001$ \\
Cesarean delivery & $1.21[1.17-1.25]$ & $<0.0001$ & $1.06[1.01-1.11]$ & 0.013 \\
Black race* & $1.61[1.51-1.70]$ & $<0.0001$ & $1.27[1.19-1.35]$ & 0.0001 \\
Pre-existing diabetes & $3.09[2.7-3.6]$ & $<0.0001$ & $2.11[1.81-2.4]$ & $<0.0001$ \\
Gestational diabetes & $1.29[1.20-1.39]$ & $<0.0001$ & $1.24[1.15-1.34]$ & $<0.0001$ \\
Hypertensive disorders of pregnancy & $1.44[1.25-1.53]$ & $<0.0001$ & $1.11[1.03-1.18]$ & 0.0035 \\
Maternal age $>40$ years & $1.25[1.13-1.39]$ & $<0.0001$ & $1.27[1.13-1.41]$ & $<0.0001$ \\
Maternal age $<20$ years & $1.31[1.26-1.36]$ & $<0.0001$ & $1.18[1.13-1.23]$ & $<0.0001$ \\
\hline
\end{tabular}

*With full term being the reference; ${ }^{* *}$ With white race being the reference.

Our results indicate that antepartum admission, admission during the early postpartum period and other factors such as extremes of maternal age, black race, pre-existing and gestational diabetes, gestational and pre-existing hypertension, preterm labor and delivery and cesarean delivery are risk factors that increase the likelihood of hospitalization between 42 - 365 days postpartum.

Our study provides novel insight regarding the risk of recurrent hospitalization up to a year after delivery following antepartum hospitalization for diabetes or hypertension-related diagnoses. Women requiring antepartum admission for management of gestational or pre-existing diabetes had significantly higher likelihood of recurrent diabetes-related admission in the first year postpartum with up to one-fifth of women admitted for diabetes with a recurrent hospitalization up to one year postpartum.

Prior studies have documented increased risks for hospitalization up to 60 days postpartum among women delivered by cesarean and those with histories of psychiatric disorders, hypertensive disorders, major puerperal infection and postpartum hemorrhage [5]-[8] [11]-[13]. However, few studies [14] have examined the risk of admission more remote from delivery. Our study provides novel insight into a select, under-studied population of women admitted within the first postpartum year but after the traditional puerperium when obstetrical deliveryrelated complications in the first six weeks may influence admission. Although we do not have data on hospital admission for this select group of women beyond one year postpartum, we theorize that these women may have comorbidities that increase the risk of recurrent hospitalizations and may be appropriate targets for closer outpatient follow-up.

Similar to other studies [6] [8], we found that the leading cause of readmission in the late postpartum period was biliary disease. Biliary disease has been found to be a major cause of postpartum non-obstetrical hospitalization with up to $0.5 \%$ of all women affected by biliary disease up to 1 year postpartum [14]. In this study, risk factors for postpartum biliary disease included obesity and ethnicity [14]; the authors demonstrated a strong association with antepartum diabetes on univariate analysis which became non-statistically significant after adjustment for obesity. We did not have maternal body mass index available for analysis; while obesity likely contributes to late postpartum readmissions for biliary disease, it is unlikely to be the sole independent predictor.

The strengths of this study are the large size of the dataset and hospitalization records linked with birth records, permitting analyses of obstetrical risk factors with readmissions up to one year postpartum at any statewide hospital. While it is possible that some birth mothers may have moved out of state within the first year after birth and are therefore missing from our cohort, we anticipate this would be a small proportion of the population and assume there would be little differential in the prevalence of our predictors and outcome between women who left or stayed within California. We posit that the results are generalizable to other diverse postpartum populations. There are limitations of this study. Our results rely on administrative claims, and not on data specifically collected for research purposes. As such, some data elements may be missing and others misclassified. We do not have reason to believe that these data events would occur differentially, and thus consider them 
unlikely to affect the underlying validity of the associations we report. Additionally, the limitations of administrative claims may not adequately reflect clinical nuances of select diagnoses. Inasmuch as this was a retrospective analysis that may be subject to residual confounding effects (for example, by obesity, an unmeasured variable), we are unable to infer causality between pregnancy-related factors and late postpartum readmission; however, this was not our intent. The ability to identify risk factors associated with readmission may prove valuable to providers who care for women in the postpartum/interconception period.

An appropriate spotlight has been placed on reducing health care costs. Our data demonstrate that women with select demographic risk factors have a higher risk of hospitalization up to 1 year after delivery. Specifically, women with an antepartum admission for gestational or pre-gestational diabetes are significantly more likely to experience a recurrent hospitalization for a diabetes related indication within a year postpartum. Pregnancy and the puerperium have been referred to as the "window into a woman's future health"; [1] this association has held true for conditions such as preeclampsia and gestational diabetes [2]-[4] and early intervention for many chronic diseases has been advocated in the postpartum period. Our data indicate there are select risk factors for a late postpartum hospitalization; these associations warrant further research on whether targeted care may be impactful on reducing these admissions in the first year postpartum.

\section{References}

[1] Saade, G. (2009) Pregnancy as a Window to Future Health. Obstetrics \& Gynecology, 114, 958-960. http://dx.doi.org/10.1097/AOG.0b013e3181bf5588

[2] Smith, G.C., Pell, J.P. and Walsh, D. (2001) Pregnancy Complications and Maternal Risk of Ischaemic Heart Disease: A Retrospective Cohort Study of 129,290 Births. Lancet, 357, 2002-2006. http://dx.doi.org/10.1016/S0140-6736(00)05112-6

[3] Lykke, J.A., Langhoff-Roos, J., Sibai, B.M., Funai, E.F., Triche, E.W. and Paidas, M.J. (2009) Hypertensive Pregnancy Disorders and Subsequent Cardiovascular Morbidity and Type 2 Diabetes Mellitus in the Mother. Hypertension, 53, 944-951. http://dx.doi.org/10.1161/HYPERTENSIONAHA.109.130765

[4] Bellamy, L., Casa, J.P., Hingorani, A.D. and Williams, D. (2009) Type 2 Diabetes Mellitus after Gestational Diabetes: A Systematic Review and Meta-Analysis. Lancet, 373, 1773-1779. http://dx.doi.org/10.1016/S0140-6736(09)60731-5

[5] Lui, S., Heaman, M., Kramer, M.S., Demissie, K., Wu Wen, S. and Marcous, S. (2002) Length of Hospital Stay, Obstetric Conditions at Childbirth and Maternal Readmission: A Population-Based Cohort Study. American Journal of Obstetrics and Gynecology, 187, 681-687. http://dx.doi.org/10.1067/mob.2002.125765

[6] Belfort, M.A., Clark, S.L., Saade, G.R., et al. (2010) Hospital Readmission after Delivery: Evidence for an Increased Incidence of Nonurogenital Infection in the Immediate Postpartum Period. American Journal of Obstetrics and Gynecology, 202, 35.e1-35.e7.

[7] Lui, S., Heaman, M., Joseph, K.S., Liston, R.M., Huang, L., Sauve, R. and Kramer, M.S. (2005) Risk of Maternal Postpartum Readmission Associated with Mode of Delivery. Obstetrics \& Gynecology, 105, 836-842. http://dx.doi.org/10.1097/01.AOG.0000154153.31193.2c

[8] Lydon-Rochelle, M., Holt, V., Martin, D.P. and Easterling, T.R. (2000) Association between Method of Delivery and Maternal Rehospitalization. JAMA, 283, 2411-2416. http://dx.doi.org/10.1001/jama.283.18.2411

[9] Jencks, S., Williams, M.V. and Coleman, E.A. (2009) Rehospitalizations among Patients in the Medicare Fee-for-Service Program. NEJM, 360, 1418-1428. http://dx.doi.org/10.1056/NEJMsa0803563

[10] Herrchen, B., Gould, J. and Nesbitt, T. (1997) Vital Statistics Linked Birth/Infant Death and Hospital Discharge Record Linkage for Epidemiological Studies. Computers and Biomedical Research, 30, 290-305. http://dx.doi.org/10.1006/cbmr.1997.1448

[11] Munk-Olsen, T., Laursen, T.M., Mendelson, T., Pedersen, C.B., Mors, O. and Mortensen, P.B. (2009) Risks and Predictors of Readmission for a Mental Disorder during the Postpartum Period. Archives of General Psychiatry, 66, 189-195. http://dx.doi.org/10.1001/archgenpsychiatry.2008.528

[12] Sharvit, M., Rubinstein, T., Ravid, D., Shechter-Maor, G., Fishman, A. and Biron-Shental, T. (2014) Patients with HighRisk Pregnancies And Complicated Deliveries Have an Increased Risk of Maternal Postpartum Readmissions. Archives of Gynecology and Obstetrics, 290, 629-633. http://dx.doi.org/10.1007/s00404-014-3255-Z

[13] Stach, S.L., Liao, A.W., de Lourdes Brizot, M., Francisco, R.P. and Zugaib, M. (2014) Maternal Postpartum Complications According to Delivery Mode in Twin Pregnancies. Clinics (San Paolo), 69, 447-451. http://dx.doi.org/10.6061/clinics/2014(07)01

[14] Ko, C.W. (2006) Risk Factors for Gallstone Related Hospitalization during Pregnancy and Postpartum. The American Journal of Gastroenterology, 101, 2263-2268. http://dx.doi.org/10.1111/j.1572-0241.2006.00730.x 
Scientific Research Publishing (SCIRP) is one of the largest Open Access journal publishers. It is currently publishing more than 200 open access, online, peer-reviewed journals covering a wide range of academic disciplines. SCIRP serves the worldwide academic communities and contributes to the progress and application of science with its publication.

Other selected journals from SCIRP are listed as below. Submit your manuscript to us via either submit@scirp.org or Online Submission Portal.
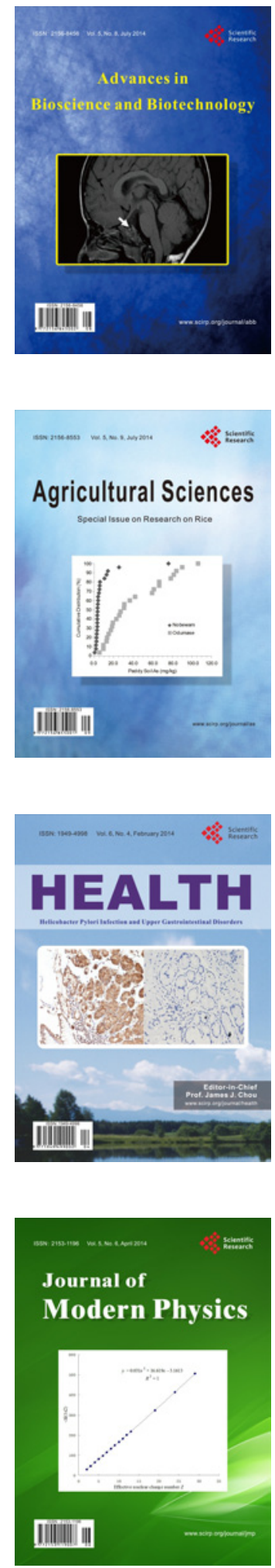
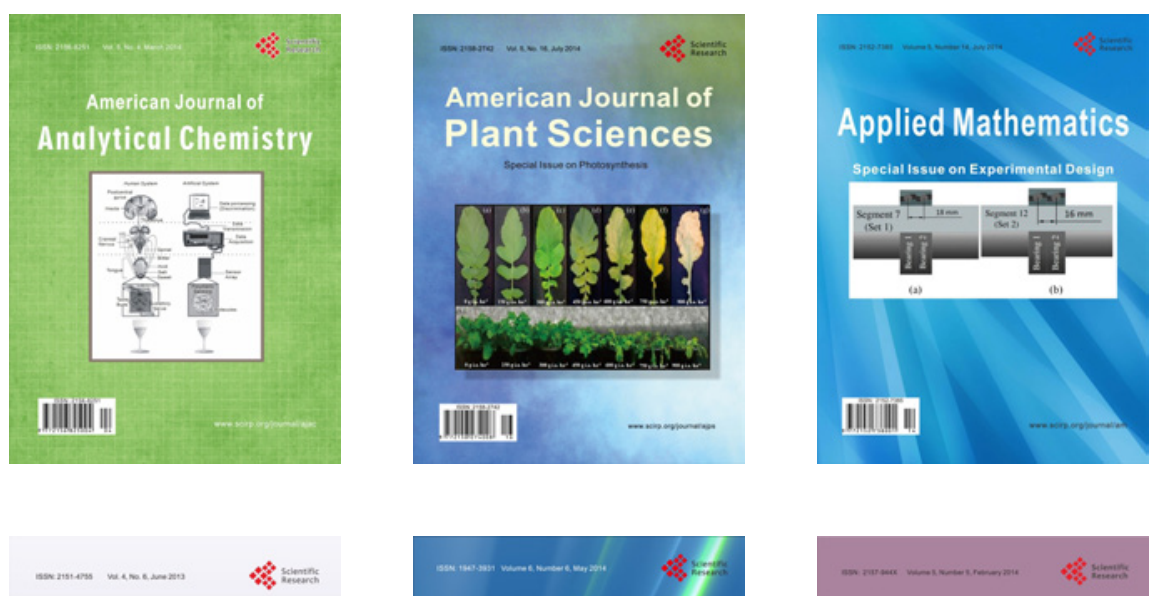

Creative Education
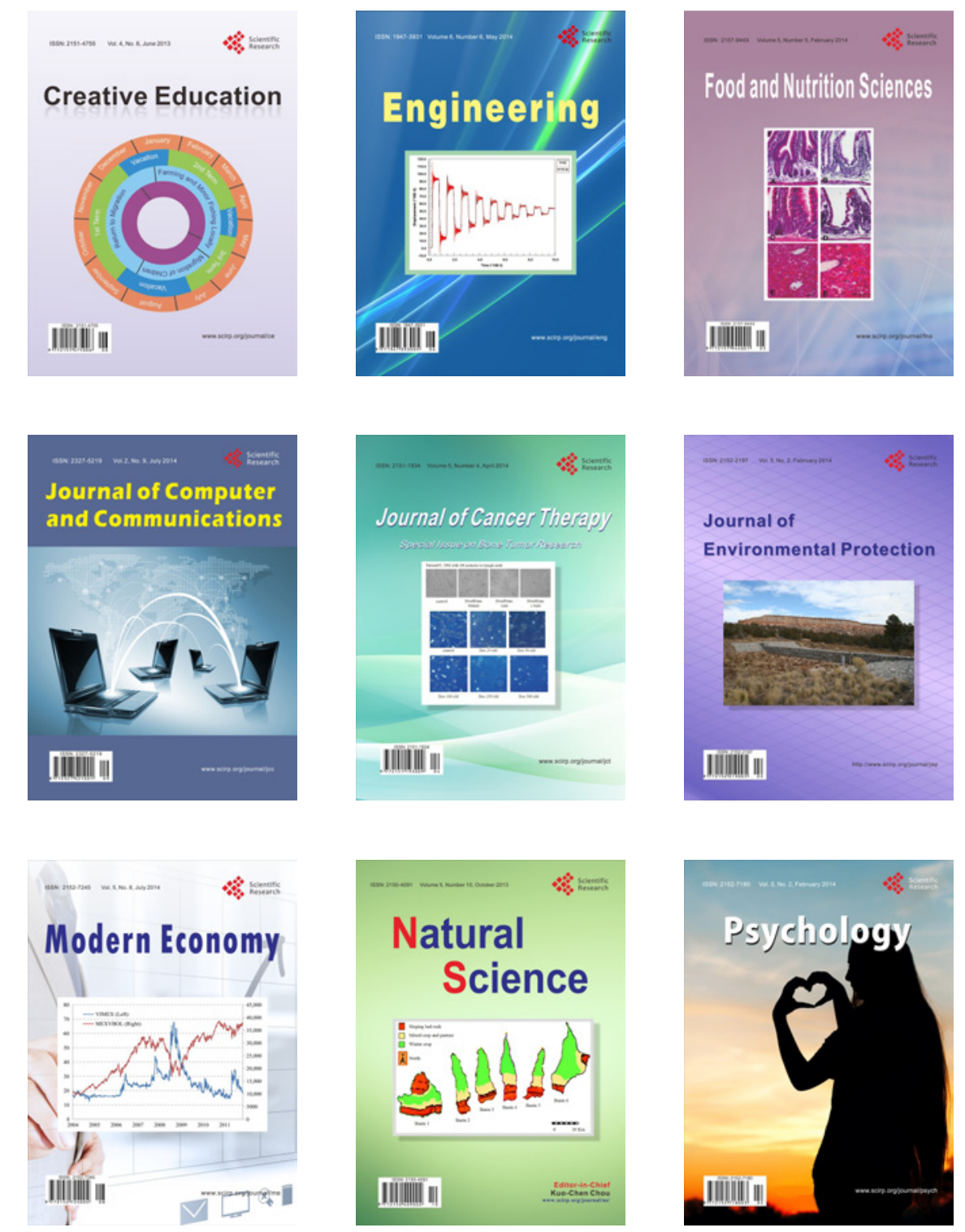